\section{Colored Apparel - Relevance to Attraction in Humans}

\section{Sullivan $\mathbf{C R}^{1 *}$, Kazlauciunas $\mathbf{A}^{2 *}$ and Guthrie $\mathbf{J T}^{2}$}

\begin{abstract}
There are numerous different dyes available, many varied fashion trends, and various different ways to change/enhance physical aesthetics. Predicting color preferences and how colors and color combinations, in a shape context, stimulate certain emotions, represents a challenging prospect. Color is a critical cue for sexual signaling, but what the preferred colors actually are in humans, is difficult to predict. Understanding color preferences and perception of color within a context such as attraction, is essential for improving color forecasting and gaining a deeper understanding of color perception. The appearance of color can change based on lighting, shape, texture, and the surrounding environment and associated colors. While these provide physical color characteristics, human vision and perception contributes to how a color appears to the individual. Perception is unique to each individual and is constantly changing due to the influence of a range of variables. This can cause someone to appear visually attractive or visually unattractive. By taking into account all the variables that contribute to human studies in color perception, tailored research can continue to be undertaken to further develop a deeper understanding of color perception and human attraction regarding visual stimulation.
\end{abstract}

\section{Keywords}

Color preference; Color vision; Color psychology; Color-fashion; Color perception; Human attraction

\section{Introduction}

The presence and use of color is universal. Although humans do not need color vision to experience motion, contour, and depth, color continually enhances and enriches society in many ways [1]. No two people see color or experience its effects in the same way, so color provokes different responses according to various situations/ associations. Predicting color preferences and how colors and color combinations, in a shape context, stimulate certain emotions represents a challenging prospect, both in a commercial context and on a more personal basis.

Color is a critical cue for behavioural tasks such as sexual signaling [1]. In many animal species, the male will use color to attract a female, common for many birds, fish and insects [2-4]. Among humans, the popular belief is that women find it more important to be physically attractive than do men. Also men seem to require both their shorterterm mates (i.e. single date, one-night stand, short-term relationship)

*Corresponding author: Sullivan CR, 325 N. Water St., Batavia, Illinois 60510, United States of America; E-mail: chelsearose.sullivan@gmail.com

Kazlauciunas A, Department of Colour Science, School of Chemistry, University of Leeds, Leeds LS2 9JT, UK, Tel: +0113-34-32939, +07872122956 E-mail: A.Kazlauciunas@leeds.ac.uk,ccdak@leeds.ac.uk

Received: July 25, 2017 Accepted: August 12, 2017 Published: August 16, 2017 and their longer-term mates (i.e. husband/wife/partner, fiancé, longterm relationship) to be physically attractive [5]. For heterosexual groupings, studies have shown that when evaluating a female's attractiveness, men focus not only on physical cues such as facial expression and body language but also on the type and color of their clothing [6].

For centuries, females have been attracted to the use of color. In the 1930's, Korda et al. [7] stated that color was particularly attractive to female cinema audiences. Yevonda, in the 1930s, stated that women were more attached to the use of color photography than were men as the medium was better able to highlight visible signs of the times, such as red hair, uniforms, flawless complexions and cosmetic combinations (lips and colored finger nails) [8]. Such use allowed females to express themselves more fully [8]. Females use color in a variety of different ways including adding beauty to their aesthetic characteristics. The use of colored make-up, accessories, tattoos, hair colorants and clothing are among ways of enhancing physical appearances. However, it is not uncommon for men to use color in certain ways to change their physical appearance, increasing their potential to attract a potential partner.

These colors and color combinations, that women see and perceive as being important in increasing their physical attractiveness, can be different to those that males find attractive. Questions have arisen concerning the role color plays in human attraction. To appreciate the significance of these questions, a basic understanding of how color is created, how the human visual system works, how the brain perceives color, what attraction is, the role of color in fashion, and what studies have been previously undertaken, is required.

Color is the visual perception of the appearance of light, interacting with matter, as detected by the eye $[9,10]$. Light is a form of energy within the electromagnetic radiation spectrum (Figure 1) [11-13]. The human visual system is capable of detecting only a small part of this spectrum, namely from $400 \mathrm{~nm}$ (violet) to $700 \mathrm{~nm}$ (red), the visible spectral range $[2,10]$.

Newton conducted an experiment in which he passed white light through a prism; the various wavelengths were refracted by different amounts (Figure 2). The dispersion of light or the spread of hues that were formed was experienced as red, orange, yellow, green, blue, indigo and violet, across the visible wavelength range (Table 1 ) $[9,13$ 16]. By combining all these wavelengths, white light is formed.

Young, Herman von Helmholtz, and Maxell refined Netwon's concept, stating that white light can be created by combining three different colors of light, the primary colors: namely red, green, and blue, or the secondary colors: yellow, cyan, and magenta $[8,14,15]$. The Young-Helmholtz-Maxwell theory states that varying combinations of the primary colors, or the secondary colors can create all natural colors.

Light can interact with materials. The light that is emitted from the object will depend on the interactions that occur between the light and the material. The experienced color of an object is dependent on three characteristics: the illumination, the interaction of radiation with matter and the ability of the radiation (visible light) to induce sensations in the eyes of observers [12]. 


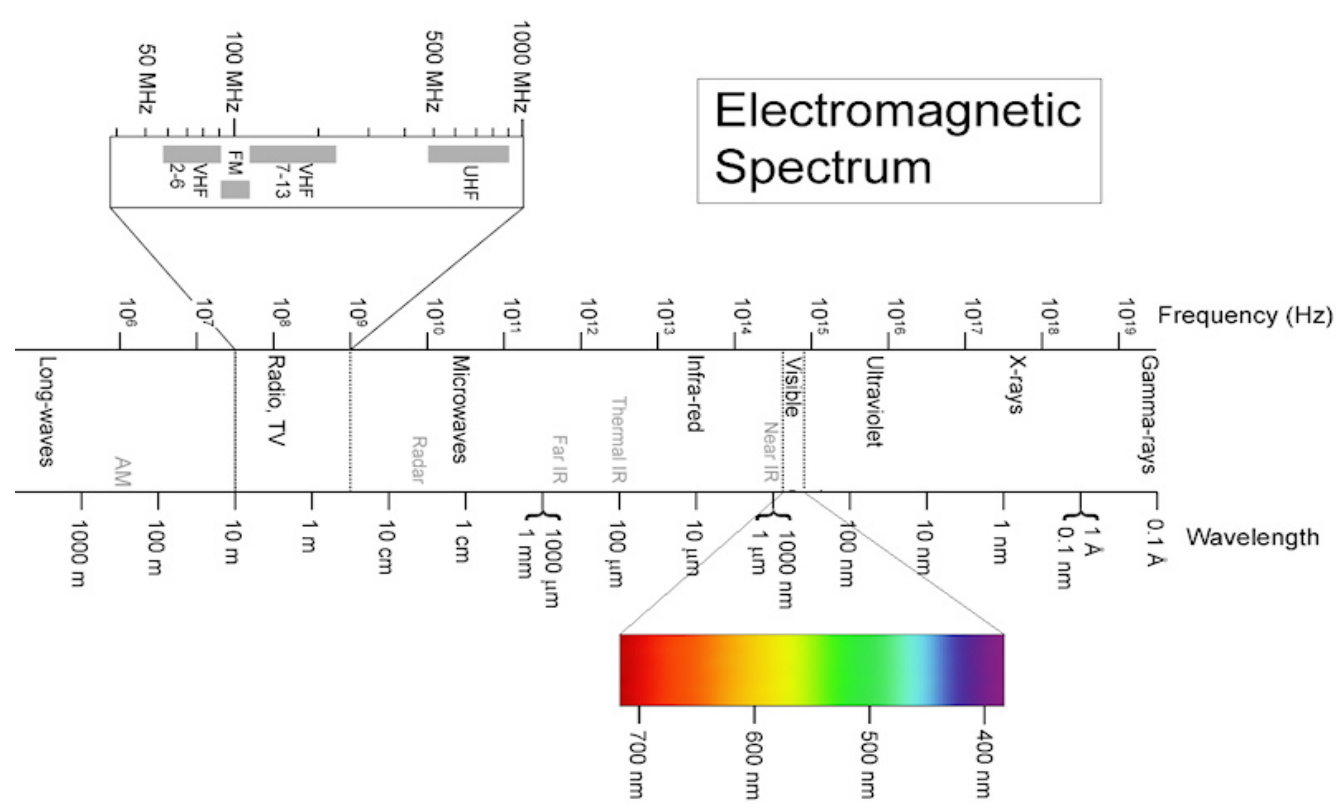

Figure 1: Representation of the electromagnetic spectrum [10]

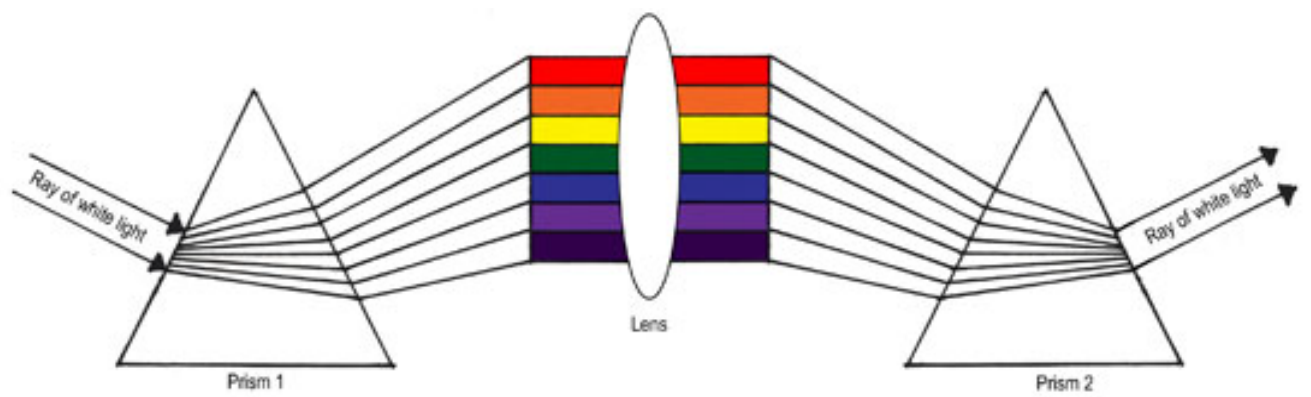

Figure 2: Newton's Prism Experiment: White light enters prism and due to dispersion, a ray of colors is formed. Recombined through the prism gives white light [12].

Table 1: Visible region of electromagnetic spectrum.

\begin{tabular}{|l|l|}
\hline \multicolumn{1}{|c|}{ Color } & \multicolumn{2}{c|}{ Wavelength (nm) } \\
\hline Violet & $400-455$ \\
\hline Blue & $455-492$ \\
\hline Green & $492-577$ \\
\hline Yellow & $577-597$ \\
\hline Orange & $597-620$ \\
\hline Red & $620-700$ \\
\hline
\end{tabular}

Depending on the type and on the strength of any illumination, objects will be perceived to possess different color characteristics. The nature of illumination is characterized by its spectral power distribution, the total amount of power that the source emits in an interval of wavelength [14]. There are many types of illumination, such as the sun, blackbody radiation, a tungsten lamp, a gas-discharge, fluorescent lamps, laser lighting and various other "artificial" light sources 1997 [12,14,15]. Different types of illuminant emit different amounts of energy, changing the way an object appears with respect to color and, in some instances, with respect to shape, depending on how the energy interacts with the material (Figure 3).

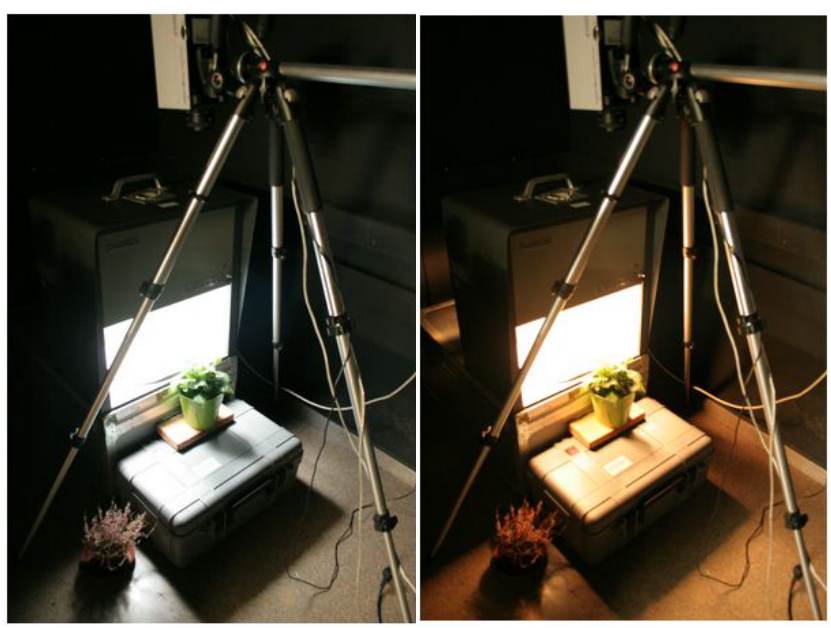

Figure 3: A photographic image under two different illuminations, CIA D65 and CIA A, each of which provides a different amount of energy [17]. *

Visible electromagnetic radiation, interacts with matter in various 
ways including absorption, reflection, interference, transmission, and scattering [10]. When light meets an object, some of the light is transmitted, some is absorbed and the rest is reflected $[11,17,18]$. Scattering is a type of reflection that occurs when the material is impenetrable to particular wavelengths $[10,14]$. The amount and direction of the scattering is dependent on the size of the particles in the material and/or any differences in the refractive indices (Figure 4) [11]. If the material is transparent, then the light passing through the matter is unchanged [10].

Depending on the wavelength(s) that is reflected from an object, the energy will trigger stimuli in the eye, being perceived as specific color sensations (Table 2). For example, green fabrics appear green because the white light that hits the fabric takes part in interactions. Red light and blue light are absorbed, whereas green light is reflected (Figure 5).

The surface finish and structure of the material can influence (increase or decrease) the scattering of the light, changing the color appearance. There are three different classified types of finish: matte, gloss, and eggshell (Figure 6). If the material is glossy, the specular reflection will be concentrated in one direction, the color being perceived as more saturated. With an eggshell finish, there is a lesser
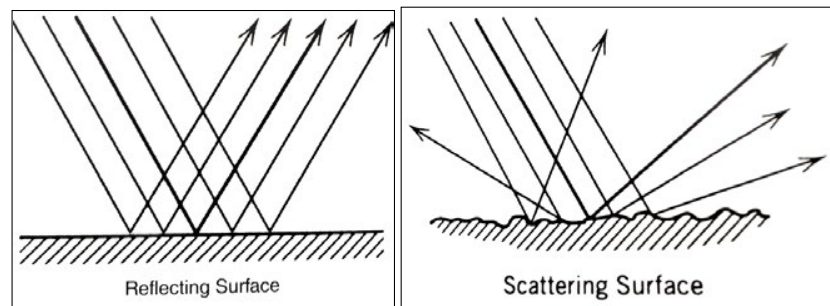

Figure 4: Illustration of light reflecting off a smooth surface (left) and off a rigid surface (right)

Table 2: Relationship between absorbed color and color transmitted/perceived.

\begin{tabular}{|l|l|l|}
\hline $\begin{array}{l}\text { Wavelengths Absorbed } \\
\text { (nm) }\end{array}$ & $\begin{array}{l}\text { Color of absorbed } \\
\text { light }\end{array}$ & $\begin{array}{l}\text { Perceived color of } \\
\text { transmitted light }\end{array}$ \\
\hline $400-440$ & Violet & Greenish-Yellow \\
\hline $400-500$ & Blue & Yellow \\
\hline $460-500$ & Greenish-Blue & Orange \\
\hline $400-620$ & Bluish-Green & Red \\
\hline $480-520$ & Green & Magenta \\
\hline $560-700$ & Orange & Turquoise \\
\hline $600-700$ & Red & Bluish-green \\
\hline
\end{tabular}

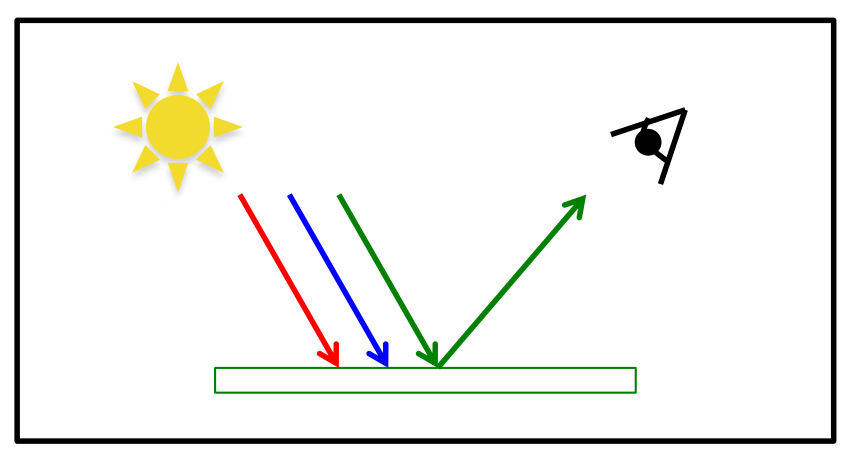

Figure 5: White light (a combination of red light, blue light, and green light) is shone onto a surface. Red light and blue light are absorbed whereas green light is reflected, making the surface appear green.*

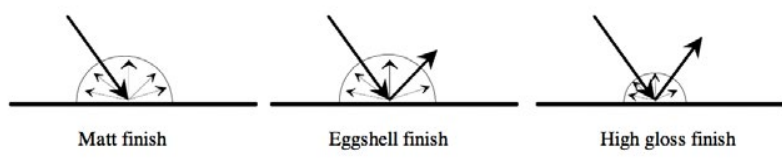

Figure 6: Spectral Reflection of three different finishes.

amount of reflection. With a fully matte finish the specular reflection is distributed over all angles. A matte finish causes a hue to appear lighter.

Light corresponds to the energy input that induces a sensation in the human visual system, giving a perceived color $[9,11]$. An object will reflect certain wavelengths, giving the material a defined 'color'.

The color of an object can be described through a combination of chromatic content and achromatic content [10]. In order to describe these attributes; an understanding of visual processes is needed. The eye is the spherically shaped apparatus that allows humans to see. A portion of light is reflected from an object and some of this light is directed into the eye [11].

\section{Color Vision Deficiencies}

Phenomena that are associated with color vision deficiencies (CVD) are of considerable relevance. Color vision provides the ability to discriminate between changes in the wavelengths of radiation that are emitted from an object $[18,19]$. The majority of humans have normal color vision [20]. The National Eye Institute states that $8 \%$ of men and $0.5 \%$ of women have a form of CVD [19,21,22]. The genes that are responsible for CVD are held on the X-chromosome. Females have two X-chromosomes. A functional gene on one of the $\mathrm{X}$-chromosomes is enough to compensate for the CVD on the other. Men do not have a second X-chromosome to make up for any loss. Therefore, men are more prone to CVD [22,23]. There are four defined categories of color vision, these being, trichromat, monochromat, dichromat, and anomalous trichromat [22-25].

Someone with 'normal' color vision is categorized as being a trichromat. Trichromacy implies that, during the processing stage, information is transmitted through a three-channel pathway, three cones [19]. When light enters the eye, all of the three cones are responsive.

Monochromatic vision is extremely rare and is present at birth. There are two classifications for monochromatic vision, cone monochromacy and achromatopsia [22]. Cone monochromacy is due to the failure of two of the three cones. Those experiencing such failure, see the world in only one hue. With this type of vision, the brain has trouble distinguishing colors, as it does not have signals from the different cones by which to compare the information. Cone monochromats also have poor visual acuity, being nearsighted. They may also have uncontrollable eye moments. Achromatopsia, arising when none of the cones possesses functional photopigments, causes people to see the world in shades of black, white, and grey $[22,24,26]$.

If one of the three cone photopigments is defective, the consequence is classified as dichromatic vision. One chromatic response is zero and there is no activation of any wavelength for the defective cone $[20,24]$. Absorbance of light by photopigments still occurs, but will fail to activate the color neural system, meaning that the defective cone will cause that hue range to be indistinguishable from grey $[20,24]$. Three different types of dichromatic vision exist: 
pronatopia, deuteranopia, and tritanopia [22]. Protanopia arises when the red cone is non-responsive, deuteranopians have no response in the green cone, and tritanopia vision gives no response in the blue cone. An example of dichromat vision can be seen in Figure 7. The more common types of dichromat are protanopes and deuteranopes, who confuse red colors and green colors [20,21].

More than $80 \%$ of the population are Anomolous Trichromats (AT) [28]. Their color vision is due to a modification in one of the cone pigments, a shift in the peak sensitivity of the cone $[20,27,28]$. Normal peak sensitivities for the small, medium, and large cones are $420 \mathrm{~nm}, 530 \mathrm{~nm}$, and $560 \mathrm{~nm}$ respectively [11,29]. For ATs, the peak sensitivities are: $475 \mathrm{~nm}, 500 \mathrm{~nm}$, and $580 \mathrm{~nm}$. Due to these peak shifts, ATs distinguish different colors from normal vision on rare occasions and sometimes are able to distinguish small color differences in a superior manner than people with normal color vision [20]. There are three types of AT, determined by which cone has the peak intensity shifted, giving protanomaly, deutranomaly, and tritanomaly. Protanomaly arises when red, orange, and yellow hues appear greener and not as bright. Deutranomaly arises when yellow and green hues appear redder. This type of observer experiences difficulty in differentiating between blue shades from violet shades. Tritanomats have difficulty distinguishing yellow shades and red shades from pink shades. Blue colors also appear greener. This type of AT is extremely rare, affecting men and women equally [22].

While people can be born with any one of these, CVDs can also be developed. Alcohol abuse, cocaine, migraines, dietary deficiencies, diabetes, glaucoma, brain damage from strokes or synesthesia, Parkinson's disease or ocular disorders can cause either short-term color vision deficiences or long-term color vision deficiencies [30-34]. The cones that are affected by various drugs and medical disorders vary. Specifically, diseases that cause lesions in the deeper layers of the retina result in a reduction in the blue-yellow responses, whereas victims of damage in the ganglion layer or the optic nerve mainly suffer from changes in the red-green senses [35]. Age can also be a contributing factor to a change in one's color vision. People above the age of fifty can develop a CVD if the crystalline lens of their eye begins to accumulate a yellow pigment that absorbs short wavelengths of light more than it absorbs at other wavelengths, skewing the perception of the short wavelength colors, (examples include blue, purple, and indigo) [31,32].

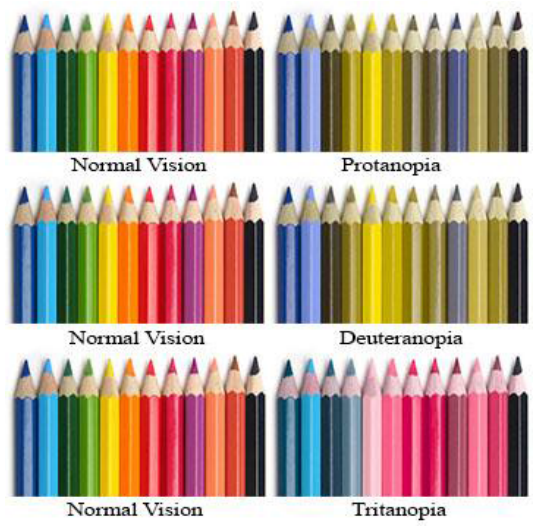

Figure 7: How eyes with the different dichromatic CVD see colored pencils [27].*

\section{Perception and Deception}

Energy that corresponds with the visible region of the spectrum is converted into a pattern of neural activity by the retinal cells. This energy is transferred to the brain through the optic nerve. The left half of each retina produces signals concerning the right half of the visual field, being transferred through the optical nerve to the left Lateral Geniculate Nuclei (LGN) of the thalamus part of the brain. The right half of each retina produces a signal of the left half of the visual system and is transferred to the right side of LGN [36]. Signals from both halves of the eye are sent to the visual cortex, where the information can be processed together.

Humans develop a perception, a certain view of the world, from these signals. Perception is the processing of sensory information in order to see, hear, taste, smell, or feel objects whilst using previous knowledge to provide a guide to one's actions with respect to such stimuli $[31,37]$. Perceptions are influenced by many factors such as emotion, values, age, culture, religion, mental state, imagination, recognition, and lifetime experiences [2].

Color specifically, influences many of the factors in life such as giving pleasure or imparting aesthetic value to an object or environment, to stimulate certain emotions, to mark territory, and to provide information [31,38]. Color is capable of detaching people from their normal environments, sucking attention into a specific location [39].

The perception of color is based on the wavelengths that are emitted, triggering stimuli. Previous experience and emotions that are attached to that specific color, together with previous experience and emotions that are associated with the object that is colored can influence one's decision. These decisions include developing a preference for certain colors and prejudices against others. Such decisions also apply to color combinations [40]. These decisions could change if colors present themselves in a continuous flux since they are constantly changing, based on the appearance of neighboring colors. Thus, humans constantly change, correct, or reverse their opinions about colors [40].

McCann created four models to describe color perception: physics, sensation, recognition, and emotion (Figure 8) [37]. The first model is based on the wavelengths emitted from the object. The second model states that color is relative to other colors in the environment, providing a sensation of appearance. The third model takes the previous experience of the viewer and the ability to have recognition into account. The fourth model considers the emotions associated with that color.

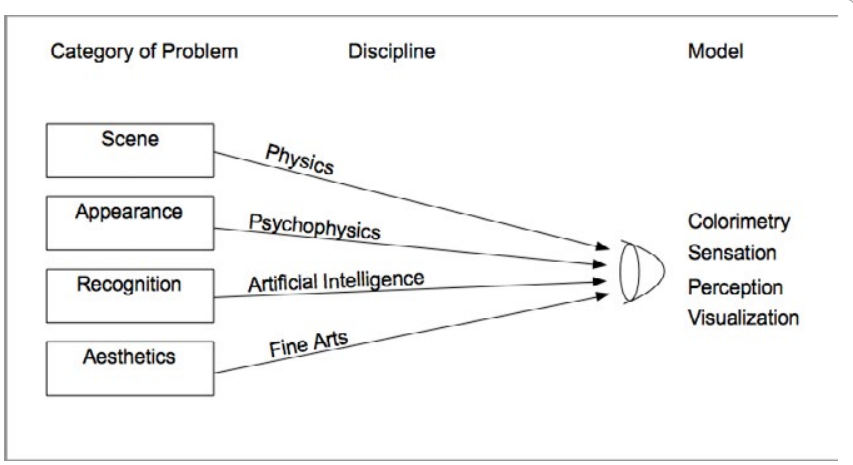

Figure 8: McCann's four models of color [37]. 
Visual deceptions do occur. It is known that not even the normal eye is fool-proof against deceptions [41]. Illusion occurs when there is a perceptual error, the sensory experience deviating from what would be expected, based on the physical characteristics of the stimulus. Illusion is created in many ways such as shading, linear perspective, aerial perspective, texture gradient, and the integration of depth information [38].

In color vision, other deceptions may arise, such as desensitization, color constancy, simultaneous contrast and metamerism, which make the colors appear differently $[10,31,37,42]$. The visual system determines the color of an object by comparing responses from the entire view. Color constancy is experienced when an object's color remains constant despite changes in the illumination and in the light that is reflected from the object towards the observer [38]. Color constancy cannot be achieved under all light sources. This gives rise to problems for operatives in many industries such as painters, dyers, and graphic printers $[10,31,10]$. Metamerism occurs when two objects are observed, with the same image/color looking the same under one type of illumination and different under another type of illumination [10]. Simultaneous contrast is the effect that arises when a color is perceived to appear either enhanced or diminished from its actual hue, due to adjacent colors, Figure $9[10,37]$.

These deceptions also play a part in the sensations that are evoked such as emotions, values, expectations and motivations. Based on these perceptions, a decision or judgment is made, followed by some action [2]. The action could be accepting or sensing repulsion of the object or person.

Several factors can have relevance. These include attraction, color and fashion options, material/substrate types, aspirations of the user and the role(s) of the colored objects, apparel, decoration, interior designs, exterior designs, nature of particular occasions and so on.

\section{Attraction}

Attraction is a judgment that is formed on the basis of the stimulation of visual perception. While attraction used to be associated to being strictly heterosexual, today's society is more accepting of various gender identities and gender fluidity. Due to this acceptance there is increased the amount of different types attraction and relationships that exist. People can identity as heterosexual, homosexual, bisexual, pansexual, queer, androgynous, etc. With increased gender identities, there are a variety of types of relationships and attraction, which include heterosexual, homosexual, transgender, and bisexual relationships. These are only a few of the different types of relationships that are mentioned in depth, but others include: polygamy, polyamory, 'open' relationships, and swinging.

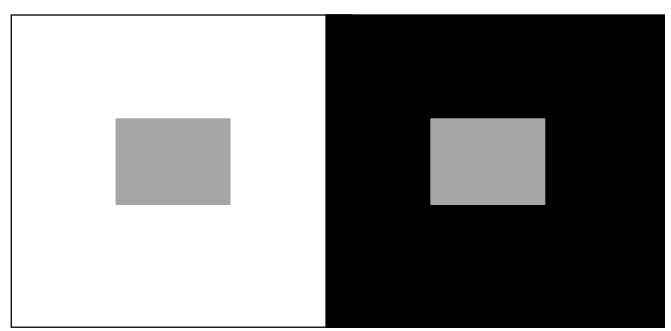

Figure 9: Illustration of simultaneous contrast.

${ }^{1}$ Color image might not be correctly portrayed depending on computer monitor settings, color vision, or how it is printed.
Sexual attraction is important, but that can change based on if the individual is in a relationship or not. Perception is influenced by life experiences and the perception of attraction could be changed based on what stage of a relationship an individual is in. There are many different stages of romantic relationships such as single and dating, in a new relationship, in a long-term relationship, newly married, married for a certain number of years, married with kid(s), single with $\mathrm{kid}(\mathrm{s})$ and dating, or divorced and dating to name a few.

When evaluating another for mate potential, attraction can arise from a range of different attributes such as physical attractiveness, personality, values, and so on [43]. The human perception of attraction is influenced by factors such as media exposure, family pressure, and status. With the use of visual perception, sexual selection can occur; a natural selection of one human over another, based on preferences of certain characteristics $[44,45]$. This selection for a potential mate is an important social decision that people make and is a prerequisite for further involvement [43].

While gender identity and different types of relationships are important to study, many researches pertaining to the use of color have been done on heterosexual attraction. Perilloux et al., believe that attractiveness is a key predictor in sexual selection [46]. Since the 1930's, studies have shown that men, across cultures, prioritize the physical attractiveness of a woman over other attributes such as liveliness, sense of humor, or exciting personality, to ensure their reproductive success [5,46-48]. Attributes that are considered physically attractive to males include those that show potential for healthy fertility. These could include being in a specific age range, appearing youthful, having a specific body shape and so on [48]. Women consider being physically attractive to be more important than men do, especially in efforts to attract the opposite sex. In order to achieve the appearance of being capable of fertility, women will actively modify their aesthetics by losing/gaining weight or selecting clothing, jewelry, hairstyles, and cosmetics. The use of color is important in improving one's aesthetic character. Women choose those colors that they perceive to be more 'seductive' [49].

\section{A History of Color in Clothing}

In Ancient times, textiles were made completely from natural hides and from natural fibers, including wool, silk, linen, and cotton. During this time sources of natural dyes were used, including roots, tree-bark, plants, animal urine and insect blood. The majority of the dyes only produced a subtle color change on these natural textiles. Also, the dyes were not particularly colorfast. The shades that were preferred included blacks, browns, greys, tans, off-whites, and reds [50]. Mainly warm colors were available during this period [51].

From the Renaissance period, the color of clothing became more important. Due to the limited dyeing technology that was available during this period, color trends persisted for decades. The color(s) developed deep symbolic meaning indicating the individuals' cultural learning, place in the social hierarchy and economic status [52]. For example, nobles wore deep rich colors illustrating their status. It was not just the color that portrayed one's status, but the silhouette and fabric were also seen as important [50]. Up until the mid-1700's men and women shared the same color palette for clothing. At which point, men began wearing more subdued and darker colors, while women wore brighter, lighter, and clearer colors such as yellow shades [51]. During this period, there was an increased consumer base driving a movement for the development of increased and better quality colors [50]. Due to this increased interest, 'fashion-seasons' appeared, 
offering softer and lighter colors for the spring and summer seasons and darker colors for autumn and winter seasons [51].

In 1856, William Henry Perkin accidentally made the first synthetic aniline-based dye, termed mauveine, which was purple in color [50]. This invention initiated movement towards the development of synthetic dyes and synthetic fibers. These two inventions contributed to the development of fashion forecasting: both for color and for silhouettes. By the late 1800 s, French textile mills began issuing color swatches of dyes that were in vogue among the top fashion designers, greatly influencing ready-to-wear market trends [50].

Consumers have modified their shopping procedures, namely how they shop, where they shop, and how often they shop. People buy more items and shop more frequently causing them to be more demanding, desiring an increase in styles, colors, and deals [53]. With the introduction of global sourcing, a selection of over 6,000 dyes has become available, giving a window for this 'fast fashion' market. Thus, consumer expectations of new designs became more ambitious. Currently it seems that a demand for companies to produce the 'right' color for each season has arisen [52,54]. Four or five color options or palettes emerge in the market at the same time, indicating the need to anticipate the diversity that develops across generations, cultures, religions, gender fluidity, and ethnic groups [52,53]. The colors chosen in business are based on how they can translate into selected merchandise [53]. Quicker changes in color cycles have caused there to be a lack of symbolic associations among colors [52]. These color cycles are now linked to many different factors such as natural trends, calendar date/year influences, and economic times. For example, in western societies the shades red, pink and black are popular for Valentine's Day [50]. In attempts to consolidate color trends in fashion, many studies have been and are being carried out to explain/ rationalize color preferences.

\section{Previous Studies of Color Preference Phenomena}

Many studies have been carried out on color preference phenomena, in which the aim was towards determining population preferences. Jastrow carried out one of the earlier documented studies of color preferences, in 1893, at the World's Columbian Exposition in Chicago. 4556 visitors were asked to vote for their single favorite color out of a set of 12 paper rectangles varying in their color. The more preferred colors were blue, red, light blue and blue-violet. The less popular colors were orange, yellow-orange, and red-orange [1].

In another study by Eysenck, only six colors were used [55] The order of preferred colors by both sexes were ranked blue $>$ red $>$ green>violet. Men and women disagreed on the order of yellow and orange. Women preferred yellow, whereas men preferred orange. The exact hue, chroma, lightness, and brightness were not classified in the samples that they used, in the study causing the results of these color preferences to be less significant.

Later studies were conducted on the basis of the Munsell book of Color giving each stimulus a specific hue, chroma, and brightness. In a study by Guilford, more than 300 subjects were questioned [56] The results showed that greens and blues were preferred. Yellow and yellow-green were the least favored colors. Overall, highly saturated colors were preferred, blue and violet colors being preferred over colors that were more yellow or yellow-green in hue. These results have been confirmed across other studies [1,55-58].

Lind conducted a study on 138 university students to determine any preference in clothing colors [58]. Subjects were given 10 Munsell standard colors, and asked to arrange them in order of preference, if the color to be used was for an article of clothing. Like the studies used to investigate general color preferences, purple-blue and blue clothing was preferred followed by red and red-purple clothing. The least preferred clothing colors were yellow-red and green-yellow.

With respect to color preference and sexual attraction, red has been by far the more studied color. The color red carries the meaning of sex and romance in heterosexual interactions in society, being represented in this way in Greek mythology, in novels, in advertising, and in the cinema, making it an aphrodisiac [59]. Anthropologists' studies found that red ochre was often associated with female fertility and was a symbol of love [60]. Red is the most powerful of the chromatic hues for attention and is known to stimulate, excite, and heighten nerve impulses and the heart rate $[39,54]$, specifically, arousing emotions that are associated with behavior signals such as sexual attractiveness $[1,59,61-64]$.

Also studies of responses to the red shade have been carried out using non-human primate species, such as baboons and chimpanzees. A display of red on the face, chest or genitalia of female primates has been shown to have an essential role in sexual-signaling/sexualreceptivity [65]. During sexual receptivity, the female primate's skin becomes redder in hue due to an elevation of estrogen, which increases the vascular blood flow under the skins' surface, causing it to exhibit an enhanced red coloration [66]. Male primates take this as a signal for mating [1,67]. Elliot and Niesta state that, due to evolution, men have a biological predisposition for the color red being a sexual-signal, indicating that men automatically activate the sexual content in their memory, at the sight of appropriate red objects/regions $[59,68]$.

Elliot et al. initiated the theory that the color red enhances men's attraction towards women, suggesting that sexually receptive women are more attractive and desirable $[69,70]$. Social psychology research concerned with investigating the effect in different situations such as lipsticks, backgrounds, colored shirts. Interest has even been expressed in the clothing used in women's internet dating images and in the response to hitchhikers $[59,60,64,71]$.

One of the studies concerned four different experiments on men who did not have a color deficiency, assessing their attraction to women who were in a red environment. Two photographic images were placed in front of the male subjects, the same women being located in front of two differently colored backgrounds. The women were black shaded and also white shaded. The rest of the photograph was colored. In the two photographs, one of the backgrounds was in red and the other was changed for each study; through white, gray, green and blue. Observers were asked a series of questions, to rate the apparent attractiveness of the woman. The results showed that that man rated women to be more attractive in front of a red background versus a white background, gray background, green background or blue background [59].

In 2013, Elliot and colleges conducted another study dealing with the choices of colored clothing that women would select when being told to interact with an attractive male and also with an unattractive male [70]. Women had the choice of wearing the same style of longsleeved shirt either in red, blue, or green. When the women were expecting to interact with an attractive male, they tended to choose the red shirt.

In today's society, there is increased interest in the use of websites 
and "apps", such as Tinder, Bumble, Match.com, etc. to attract a significant other. As in meeting in person, appearance is the first experience of dating sites. Gueguen and Jacob carried out a study that was focused on women's personal advertisements that were displayed on meeting sites. The results showed that women received more interest from men when the clothes in the chest region were the color of red rather than shades of black, white, yellow, blue or green. Men showed more sexual interest in women wearing red, the color encouraging the man to make contact with the woman [60].

\section{Limitations of Human Studies}

Research into color preferences is challenging. The design, execution, and interpretation arising from perceptions are particularly difficult to rationalize [52]. Humans are extremely complicated beings, each person having his/her own perception of the world. Many factors can "interfere" with the conduction of a study of human perception and human preferences, including internal factors and external factors, covering age, sex and gender identity, type and stage of any relationship, culture, religion, and geographical location. All of these factors influence the quality of data obtained.

\section{Internal/external factors}

Many factors influence one's perception. Butts stated that observers are affected by internal factors and external factors, each of which contributes to inconsistencies [38]. Some internal factors that were not accounted for include fatigue, emotional state, the influence of chemical substances, and personal bias. External factors include previous experiences, conditioning, and the environment. In studies involving humans, it is impossible to account for the individual differences between people, such as in their positive reactions and their negative reactions to certain colors chosen by parents for their child. To provide better understanding of preferences, studies should deal with broad cultural preferences. Controlling as many aspects of the study as possible makes the results stronger. Factors that can be controlled include lighting, distance, sample types, location, age of participants, gender, sexual orientation, stage of the relationship, question types and their sequencing.

\section{Age}

Ethical considerations play an incredibly important roll if and when age is involved. These can limit a study. From an ethical standpoint some questions are considered to be too personal or offensive to certain age groups. For example, asking the participants the gender to which they are sexually attracted, whether or not they are currently on drugs or intoxicated, whether or not they have some medical condition, is not considered to be appropriate. Questions need to be thoroughly thought out in terms of the age group of potential respondents. Also the questions need to be ethical and the wording appropriate for the age group. The questions should be relevant to the study objectives. To require meaningful results, the questions need to be asked in such a way that they are fully understood and interpreted by the respondents. Using age appropriate words might be necessary. Clearly if a participant does not understand the questions, the results will be skewed.

Color preferences and perceptions change with age and with experience. The duration of the research study can influence the outcome. By gathering information from a variety of different age groups and different genders, a better understanding of color preferences can be gained. It is known that brighter colors are attractive to children, whereas softer shades are generally more attractive to older consumers [54]. However, many groups are less concerned about being type cast or judged on the basis of the colors they wear. Color is becoming more of a form of expression. Social changes in terms of age and color add complications. By observing groups of people over a long period of time, information concerning the ways that individual preferences for color brightness changes with age, could be analyzed.

\section{Geography, culture, and religion}

A person's perceptions and use of color are inherent, cultural and highly individual [50]. The power of color use comes from its symbolic significance. Color perception can embody a variety of connections across experiences, emotions, political, status, religious affiliations, fertility, and other information types that might be difficult to convey in other forms of communication. These factors can develop over time. Brannon suggests that there is a relationship between color preference, ethnic identity and geographic area [52]. For example, in Western cultures pink is often associated with pastels and is marketed towards girls and towards use during certain calendar seasons. While in Asian cultures and Hispanic cultures, magenta and Fusia is marketed to consumers instead [50].

Colors can develop symbolic meanings that are based on religious beliefs such as power, purity, wealth, resurrection, etc. Muslims, Buddhists, Islamics, and Hindus associate the color green with meanings such as paradise, resurrection or the transformation of powers of nature, protecting all travelers and having profound connection to nature $[16,50,72]$. Red is a symbol of power for both Hindus and Buddhists. Women wear red on their wedding day if they are members of these religions, since red symbolizes all types of power, including sexual power. Christian women usually wear white on their wedding day, to indicate their purity [16].

People following specific objectives use color for a variety of reasons. Yellow specifically has been used when declaring war on a certain race of people, marking unwanted people of certain religious groups. Muslims in Baghdad, tagged people that were considered 'other', such as Jews and Christians with a yellow bandage in the way Jewish people were tagged with a Yellow Star of David in Nazi Germany. When India was under Islamic rule, Hindus were also labeled with yellow armbands. Even today, the Taliban mark Hindus that are living in Afghanistan with yellow [16]. In China a man wearing a green hat represents the husband of an adulteress, making him an outcast. The rest of society would steer clear of such people. In Russia gay men are labeled using the color 'sky' blue [72]. In many countries a color can be associated with someone in a particular political party. In the USA red is worn in association with conservatives or the Republican Party whereas blue is worn if you are associated with liberalism in the Democratic Party. Political associations with colors differ across the world [72].

Cultures have developed symbolic associations with specific colors. In some cultures, color symbolizes certain emotions [72,73]. Citizens of Egypt, in mourning, wear yellow. Blue is used in Iran, red is used in South Africa, purple is used in Thailand, and black is used in America and Europe [73]. Economic and social factors can influence preferences. Cultural preferences can be overtaken by the mood of the time. Typical examples include economic conditions and events in fine art/music/TV [50]. For example, in 1901 people in England wore black for the entire year when mourning Queen Victoria's passing [51]. More recently, in 2010, South Africa won the FIFA World Cup. Therefore a color palette for sports clothing that was 
inspired by African flag colors was developed, that included yellow, red, green and black [50].

Economic changes such as the creation of a new dye can influence societies' desire for that dye. Six thousand years ago, Egyptians imported Lapis Lazuli, 'ultramarine blue', from the mountains of Sar-i Sang in Afghanistan. Due to its limited supply, it was highly valued. The pigment was used in jewelry and headdresses to indicate wealth. The color increased the value of clothing and paintings due to its rarity and expense $[16,74,75]$. The demand for Lapis Lazuli or 'ultramarine blue' was so great that, in 1824 , French society offered a prize of 6,000 Francs to anyone who could synthesize this pigment [74].

The representation of certain colors or color combinations transcends cultures. A rainbow flag is used to symbolize being 'allinclusive'. It also represents God's covenant with Noah 'never to destroy humanity again'. The rainbow flag was used to commemorate the Stonewall riots in New York, to represent Incas who were protesting against invading Spaniards and to symbolize Jewish territory in Far East Russian from non-Jewish neighbours. Today the rainbow flag is a symbolism of Gay pride all over the world [72].

Immigration events can influence a colors' popularity within a specific geographical region [52]. Between 2014 and 2015 630,000 people immigrated into the United Kingdom [76]. Such immigration is occurring worldwide. These large groups of people do not include refugees coming into a country without legal immigration paperwork. Such large groups of people influence developing cultures. Immigrants from all over the world bring their own culture, color influences and religions. These ultimately, affect the popularity of the colors by consumers.

For any assessment to be meaningful, data needs to be collected from a wide variety of locations with many participants, if one is to acquire a significant understanding of color perceptions and color preferences.

\section{Factors Influencing the Color-fashion Combinations Fashion and individuality}

Color can be used convey messages and signals. Colors grab attention and are capable of "sending" messages that alter perceptions $[50,77,78]$. Fashion includes all aspects of visual appearance: clothing, jewelry, hair, body, skin and general decoration. Each of these aspects provides a specific communicated cultural identity [79]. Whether society likes it or not, the color, style and texture of personal aesthetics express a position with respect to gender, ethnicity, race, class, nationality, religion, sexuality, and age. People use color to express visually their social identity, hierarchy, emotions, political leanings, personal identity, self-image, and esthetic tastes $[49,73,78,80]$. Color conveys values, attitudes, personalities, and tendencies towards conformity or individuality $[78,79]$.

Some fashion styles and colors provide ways of group identity. They provide a guide to the roll one plays, or skills one has. They also indicate with whom one is associated with professionally, socially, nationally, religiously and in heritage [78]. For example, colors are used to associate individuals with a certain team in sports. It is known that people who are close in age and share similar interests tend to dress alike. This helps provide individuals with confidence, psychological approval, comfort, and acceptance [78].

Although each person is unique having one-of-a-kind features such as a specific hair color, eye color, skin color and unique markings, these are not always sufficient to provide "well being". Individuals attempt to improve their appearance using various beauty technologies in order to attract a favorable response or attention [78]. Men are thought to have a predilection for novelty. Therefore, women are considered to be more likely to remake their identity constantly in response to this [81]. Society accepts male imperfections more readily than it does those of women [77]. The invention of various beauty technologies has allowed women to emphasize or construct sexual attributes so that they appear more visually stimulating, hiding imperfections, when and where possible $[81,82]$. Imagination modification occurs in all cultures. However, practices are specific to each habitus. In certain cultures, body decoration is a purposeful behavior moderator, signifying specific characteristics such as fertility, sexual availability, strength, religious affiliation, status and power [82]. Western postmodern culture is thought to encourage narcissistic individualism, making body modification and maintenance an essential part of self-expression [79]. There is a constant demand for self-improvement and for the use of additional adornments in order to construct a unique identity $[80,81,82]$. People treat their bodies in a personalized way, altered for their pleasure [83]. No one in excluded from being able to use beauty technology.

The use of modifications offers the possibility to emphasize or to play down certain features. People make transformations both temporary or permanent, to project their own desired self-image [82]. There are many different ways to change appearances permanently. These include cosmetic surgery, reconstructive surgery, tattoo art, bleaching of the skin, hair plugs, face lifts, Botox use and dental work [81]. Cosmetic surgery can be used to change the shape of the human form, for example by implanting poly (siloxane) or related materials into certain areas of the body such as breasts, lips, posterior and cheeks. This type of surgery changes one's appearance into a form that nature never intended. Reconstructive surgery is used to make permanent changes to the body for health reasons, such as breast reductions, providing better airways in the nose, liposuction and restoring one's appearance [77]. These changes can result in a noticeable shape change in one's appearance. Routes to the permanent change of one's appearance include attempting to change skin color or skin imperfections. Moles may be removed and scars hidden. Other routes involve simply decorating the skin. Tattoos are a very popular way of personalizing the body, through art [83]. This is a way of creating very personal and unique designs. No two tattoos appear the same. Tattoos add color and a design to the skin. They are capable of permanently putting cosmetics onto the face and of hiding scars.

Currently a disposable society exists. Permanent changes cause concerns, whereas temporary or semi-permanent changes are more appealing. There are several temporary changes that can emphasize various body features. These include the use of hoopskirts, bustles, corsets, brassiers, cosmetics, haircuts and hairstyles, and nail polishes [81]. The most available form of temporary beauty technology is cosmetic. Cosmetics have been used for centuries to enhance features, distracting from the less desirable features [49,81]. For example, cosmetics can be used to help reduce the visual appearance of wrinkles, giving the illusion of being younger. Hair coloration, such as bleaching and/or dyeing, is a relatively simple way in which one can to change visual appearance. Other changes include fake eyelashes, lip plumping, curling/straightening hair, hair removal, facials, fake tattoos, teeth whitening, wearing colored contact lenses, losing weight and gaining weight $[49,81]$. 
Color is essential to visual appearance. While some changes to visual appearance are easier to achieve than others, changing the color and style of clothing, jewelry, and accessories is the more common. People inherently have a blue or yellow undertone to their skin. Selecting a color pallet that accentuates these features makes someone appear more visually stimulating. Wearing an inappropriate color can cause a person to appear unnatural. People from Asian, India, Africa, South America, Scandinavia and some Northern and Southern Europeans have blue undertones to their skin. Some people look best in silver jewelry and vivid colors. Some Scandinavians, British, Northern Europeans, and Irish have yellow undertones to their skin, causing them to appear better in warmer colors. Dark colors tend to drain their skin tone, making them look paler [78]. Changing hair color or eye color can cause someone to appear unnatural, as in the use of a blue hair dye or a purple hair dye.

Image modifications and beauty technologies transcend the barriers of sex, age and class [83]. They can give the illusion of making someone appear taller, shorter, larger, smaller, younger, prettier, and so on [78]. Beauty technology is used to give visualization, leading to greater acceptance into society, providing one with ways of expressing oneself, showing how one feels and indicating views and values [81]. People can change their image to appear more or less prestigious, intelligent, beautiful and religious, also showing the acceptance or rejection of certain social 'norms' or groups.

\section{Fashion and availability}

In a 'fast fashion' driven society, there is a continuous movement towards buying into new fashion trends. Some consumers are interested in styles that are new and innovative, creating their own unique image. Others like to imitate royalty and celebrities [50]. Although there may be interest in particular styles and colors, those styles and colors may not be easily obtainable.

People have used fabric to make clothing for thousands of years. As societies and technologies progressed, people were able to purchase completed garments. The evolution of the sewing machine, in 1846, moved fashion from being a hand-made clothing technology to mass-produced ready to wear clothing $[50,80]$. This not only made clothing more affordable, but also allowed for a wider variety of styles to be accessible to mass markets. Shopping styles have consistently changed. Out of town shopping centres, outlet and shopping precincts (malls), one-stop shopping stores and online shopping have opened up opportunities and choices to consumers [53].

Internet shopping has dramatically increased accessibility to those consumers that have far commute to a store, those who cannot drive, those who have physical impairments restricting their ability to go into a store and those with busy lifestyles. The Internet is an easy to use interface that offers a wide variety of delivery options [53]. These have made shopping somewhat easier and less frustrating, especially during holiday seasons.

Some consumers want specific clothing items in a specific color. Historically, legislation has been put into place to ensure that certain color(s) were only to be worn by the upper classes and royals. Medieval Europeans developed sumptuary laws that allowed only the nobility to wear costly colors such as purple that was extracted from Mediterranean seashells. These defined those who could wear certain colors, the amount of color allowed and when the color could be worn [16,73].

\section{Fabrics and color appearance}

Texture: Appearance is defined as the observers' response to the combination of both the color and the physical characteristics [38]. Color is only one aspect of the total appearance. Many other phenomena such as the physical characteristics, contribute to the overall appearance. The characteristics of relevance to fabric include construction, width, weave, surface gloss or matte surface, or factors that alter the way the sample interacts with light [11]. Several other factors affect the color appearance of clothing. These include the fiber type, fabric structure, texture, and finishes [11].

Texture relates to the surface quality of goods, such as the roughness, the smoothness, the softness, the stiffness, the dullness, and the glossiness [78].

Color application: There are various methods of applying color to fibers, to yarns, or to fabrics. These include bleaching, dyeing, printing and other embellishments. Bleaching and dyeing can occur during any point of the construction development, starting at the fiber pre-yarn, in the yarn form, and after the yarn has been constructed into a fabric [84]. Bleaching is a chemical process in which the natural color is removed prior to the dyeing to ensure final products' applied color is 'true' or to create 'whiter' goods [78]. If bleaching is not used prior to dyeing, applying the dye, the "ground" color influences the final color appearance [85].

Dyeing is the method of giving color to fibers, yarns and fabrics $[78,84]$. No individual dye is best for all textile fibers. Dyes have different physical properties such as absorbency and acidity/basicity. The choice of dye depends on the fiber(s) used and the results desired [78]. Mixing fibers can create superior final properties. However, problems arise when dyeing such fibers because the different fibers have different physical properties and chemical properties. Cellulosic fibers can be successfully colored using reactive dyes, direct dyes and vat dyes. Protein-based fibers require a bonding agent, being better colored using vat dyes, acid dyes, and mordant dyes. Synthetic fibers vary depending on their composition. Polyesters can be successfully colored using disperse dyes. Spandex can be dyed using metal complex acid dyes [84]. There are risks to dyeing fabrics, such as uneven dyeing, shade variation, dye spots, and possible poor color fastness [86]. According to Rigg, the exact color is not as important as the uniformity of color [86]. When judging color, some sort of averaging takes place. Thus, the observer is conscious of any nonuniformity. Any appreciable difference in color on the different parts of a standard can influence the perception of the color. Treatments and finishes applied to fibers, yarn, and fabrics are used to enhance physical properties.

Finishes and treatments, both mechanical and chemical, can be applied to garments to improve their appearance, their feel, and/or their performance [78]. The treatments or finishes can be applied prior to dyeing or after the dyeing process. They may make the least desirable characteristics of the fabric more acceptable. Chemical treatments usually affect the performance of the garment. Examples include making fabrics antistatic, crease-resistant, moth-resistant, flame-resistant, mildew-resistant, easy soil release, waterproof, stain-resistant, or having controlled shrinkage. Mercerization is a chemical process that increases the strength, the absorbency, the affinity for dyes, and the luster of cellulosic fibers, enhancing the color appearance. Mechanical treatments affect the "size" and appearance of the fabric by altering the surface. Cutting, drying, stretching, and compressive shrinking, are all ways of correcting the width and the length of fibers, yarns, or fabrics. Brushing/napping, calendaring, and singeing can influence the visual texture, the softness, the smoothness, and the fuzziness of the fabric. 
Other ways of adding color and design to fabrics include printing and embellishment. Various methods such as screen-printing, digital textile printing, stencil printing, or machine printing are used to create patterns like cheetah, floral prints and so on, onto fabrics. Such methods are also used to place company logos or brands onto clothing items. Beading, embossing, embroidery, rhinestones, sequins, and appliques are ways of embellishing an item or personalizing an item [84]

Appearance: Color depends on how light is reflected, absorbed, or scattered from a surface, each being relevant to garments. Depending on the interaction between light and surfaces, illusions can be created, emphasizing certain aspects of the object or body. Color can create an illusion that a person is shorter, taller, thinner, broader and can emphasize certain areas of the body. Textile surfaces have visual attributes such as being glossy, sheer, bright, textured, and so on. Garments based on bulky, heavy, fuzzy, or shaggy textiles add visual size when worn, disguising figure irregularities. These rough surfaces also subdue colors because the light hits the uneven surfaces at different angles, causing the light to scatter, making the color appear duller. Shiny textures reflect more light off the body making the color appear lighter and brighter, causing the body to appear larger as it emphasizes body contours. Dull textures absorb more light making a person appear smaller. Sheer fabrics reveal true body shape because the skin of the wearer can be seen through the fabric, which also causes the color to be subdued.

Added visual texture can create illusions that affect the visual size of the wearer. Horizontal lines can make a person appear shorter and wider, whereas vertical lines give the illusion that someone is taller and thinner. Large bold patters and sharp contrasts between colors emphasize the location where they are used. This can help enhance specific features, such as highlighting the face, enhancing chest size and defining a waist. Small, subdued, overall prints tend to give the illusion that the wearer is smaller. Prints have the ability to hide structural features of garments such as seam lines, darts, buttons and zippers [78].

The texture, color/print and style influence whether or not the fabric is visually attractive. Fabrics become garments that individuals will wear. The shape and coloring of the individual influences how colors are reflected and seen by another person. Color/print, style, and texture can enhance a person's attractive features and can also hide their less desirable attributes.

\section{Conclusions}

Color is critical to creating attractiveness or unattractiveness. In today's society both genders use color to enhance their visual, aesthetic appearance. There are temporary beauty technologies and permanent beauty technologies. These allow someone to completely transform their physical appearance. These include plastic surgery, tattoos, and cosmetics. One way of enhancing one's appearance is to change clothes, including the color of clothes. Clothing provides people with ways of expressing individuality, conformity, beliefs, religion and so on. The use of color has become an important expression of who we are, how we feel and what we believe.

The lighting conditions an individual's color vision attributes, the texture, the presence of surrounding colors and personal experiences cause colors to appear differently. In today's society choosing colored clothing is challenging. Values, customs and restrictions are associated with certain colors, affecting the way colors are experienced and understood [79]. Colors establish attractiveness, class, occupation, beliefs and so on [16]. Understanding the connotations that a color carries varies from culture to culture, among different social groups, and individuals [84]. Thus research on human preferences is challenging. When researching those color preferences that pertain to sexual attraction, there are many challenges. Perceptions are influenced by age, gender, identity, sexual orientation, geography, culture, religion, economic times, stage of relationship, and personal upbringing. The unpredictability of preferences is influenced by personal experiences [84]. While there are these challenges in research, studies concerning the influences of color on sexual attraction have been conducted $[86,87]$. However, there is still much research to be done.

\section{References}

1. Hurlbert A, Ling Y (2012) Understanding colour perception and preference. In: Best J, ed. Colour Design: Theories and Applications. Woodhead Publishing Series in Textiles Oxford: 129-157.

2. Guthrie JT (2003) Psychology and Perception of Colour and Shape. Surface Coatings International Part B: Coatings Transactions 86: 35-42.

3. Houde A (1997) Sex, color, and mate choice in guppies. Princeton University Press, Princeton, USA.

4. Andersson M, Simmons L (2006) Sexual Selection and mate choice. Trends Ecol Evolut 21: 296-302.

5. Feingold A (1990) Gender Differences in Effects of Physical Attractiveness on Romantic Attraction: A Comparison Across Five Research Paradigms. Journal of Personality and Social Pyschology 59: 981-993.

6. Dunn MJ, Doria MV (2010) Simulated attraction increases opposite sex attractiveness ratings in females but not males. Journal of Social, Evolutionary, and Cultural Psychology 4: 1-17.

7. Korda A (1937) They Talk Colour. The Journal of the Association of CineTechnicians 3: 192

8. Smith L (2002) 'There is a Garden in her Face': Madame Yevonde's Photographic Colour. Women: A Cultural Review 13: 121-139.

9. Tilley R (2011) Colour and the Optical Properties of Materials: An Exploration of the relationship Between Light, the Optical Properties of Materials and Colour. ( $2^{\text {nd }}$ edtn), John Wiley \& Sons Ltd, West Sussex, UK.

10. Kuehni RG (2013) Color: An Introduction to Practice and Principles. ( $3^{\text {rd }}$ edtn), John Wiley \& Sons Inc., New Jersey, US.

11. Xin J (2006) Total colour management in textiles. ( $1^{\text {st }}$ edtn), Woodhead Publishing Ltd, Cambridge, UK.

12. Sinclair RS (1997) Light, light sources and light interactions. In: McDonald, R. ed. Physics for Industry. ( $2^{\text {nd }}$ edtn), Society of Dyers and Colourists, Bradford, England.

13. Armstrong T (1996) Colour Perception: A Practical approach to colour theory. Tarquin Publications, Strabroke, Australia.

14. Malacara D (2002) Color Vision and Colorimetry: Theory and Applications. (2nd edtn), The Society of Photo-Optical Instrumentation Engineers, Bellingham, US.

15. Nassau K (2001) The Physics and Chemistry of Color: The fifteen causes of color. ( $2^{\text {nd }}$ edtn) New York: John Wiley \& Sons Inc, New York.

16. Eckstut J, Eckstut A (2013) The Secret Language of Color: Science, Nature, History, Culture, Beauty of Red, Orange, Yellow, Green, Blue and Violet. Black Dog and Leventhal Publishers, New York.

17. Image and Signal Processing Group. 2015. IPL Calibrated Color Image Database: Natural Objects under CIE D65 and CIE A.

18. Skorupski P, Chittaka L (2011) Is colour cognitive? Optics \& Laser Techonology 43: 251-260

19. Valois K, Valois R (2011) Color Vision. In: Valois K. ed. Seeing. Academic Press, San Diego, California.

20. Hurvich L (1981) Color Vision. Sinauer Associates Inc, Sunderland.

21. Best J (2012) Colour specification and visual approval methods for textiles. In: 
Best J. ed. Colour Design: Theories and Applications. Woodhead Publishing Series in Textiles, UK.

22. National Eye Institute (2015) Facts about Color Blindness.

23. Neitz M, Neitz J (1998) Molecular Genetics and the Biolgical basis of Color Vision.

24. Peddie W (1922) Color Vision: A discussion of the leading phenomena and their physical laws. Edward Arnold \& Co., London.

25. Oblath O (1929) International Labour Office: Studies and Reports-Series F (Industrial Hygiene). P.S. King \& Son, Ltd., London.

26. Snowden R, Thompson P, Troscianko T (2006) Basic Vision: an introduction to visual perception. Oxford University Press, Oxford.UK.

27. Colour Blind Awareness CIC. 2015. What is colour blindness?

28. Ro Y, Yang S (2004) Color Adaptation for Anomalous Trichromats International Journal of Imaging Systems and Technology 16: 16-20.

29. Sharpe LT, Stockman A, Jagle H, Nathans J (1999) Opsin genes, cone photopigments, color vision, and color blindness. Cambridge University Press, UK

30. Desai P, Ray M, Roy A, Brown S, Smelson, D (1997) Impaired color vision in cocaine-withdrawn Patients. Arch Gen Psychiatry 54: 696-699. https://www. ncbi.nlm.nih.gov/pubmed/9283503

31. Blake R, Sekuler R (2006) Perception. (5 $5^{\text {th }}$ edtn), McGraw-Hill, Boston, US

32. Beirne R, Mcllreavy L, Zlatkova M (2008) The Effect of Age-Related Lens Yellowing on Fransworth-Munsell 100 Hue Error Score. Ophthalmic Physio Opt 28: 448-456.

33. Shepherd A (2005) Colour Vision in Migraine: Selective Deficits for S-Cone Discriminations. Cephalalgia 25: 412-423.

34. Brasil A, Castro A, Martins I, Lacerda E, Souza G, et. al (2015) Colour Vision Impairment in Young Alcohol Consumers. PLoS One 10: e0140169.

35. François J, Verriest G (1961) On Acquired Deficiency of Colour Vision, with Special Reference to Its Detection and Classification by Means of the Tests of Farnsworth. Vision Res 1: 201-219.

36. Bruce V, Green P, Georgeson, M (2003) Visual Perception: physiological, psychology, and ecology ( $4^{\text {th }}$ edtn) Psychology Press, UK

37. McCann J (2000) Simultaneous Contrast and Color Constancy: Signatures of Human Image Processing. In: Davis, S. ed. Color Perception: philosophical psychological, artistic and computational perspectives. Oxford University Press, New York

38. Butts K (2006) A practical guide to Visualize evaluation of Textile Samples. In: Xin, J. ed. Total colour management in textiles. Woodhead Publishing Ltd, UK

39. Bell J (2009) Seeing the Light. Review Saturday Guardian.

40. Albers J (1963) Interaction of Color. Yale University Press, USA.

41. Richmond Products Inc. (2016) Farnsworth D-15 and Lanthony Test Instructions Rev 1.7. Richmond Products Inc., New Mexico, USA.

42. Lotto R, Clarke R, Corney D, Purves D (2011) Seeing in colour. Opt Laser Technol 43: 261-269.

43. Back M, Penke L, Schmukle S, Sachse K, Brkenau P, et al. (2011) Why mate choices are not as reciprocal as we assume: the role of personality, flirting and physical attractiveness. European Journal of Personality 25: 120-132.

44. Colman A (2015) A dictionary of psychology. Oxford University Press, New York, USA.

45. Hosken D, House C (2011) Sexual selection. Curr Biol 21: R62-R65.

46. Perilloux C, Cloud J, Buss M (2013) Women's physical attractiveness and short-term mating strategies. Pers Indiv Differ 54: 490-495.

47. Miner E, Shackelford T (2010) Mate attraction, retention and expulsion. Psicothema 22: 9-14

48. Singh D (2004) Mating strategies of young women: role of physical attractiveness. J Sex Res 41: 43-54.

49. Cash T, Dawson K, Davis P, Bowen M, Galumbeck C (1989) Effects of cosmetics use on the physical attractiveness and body image of American college women. J Soc Psychol 129: 349-355.

50. Scully K, Cobb D (2010) Color forecasting for fashion. Laurence King Publishing Ltd., London, UK.

51. Hill M, Bucknell $P$ (1987) The evolution of fashion: pattern and cut from 1066 to 1930. Batsford, London, England.

52. Brannon E (2010) Fashion forecasting. Fairchild Books, New York, USA

53. Littlewood G (2006) Effective colour communication from mind to market. Total colour management in textiles. Woodhead Publishing Ltd, Cambridge, UK

54. Eysench H.J (1941) A critical and experimental study of colour preference. Am J Psychol 54: 385-394.

55. Guilford J.P (1959) A system of color-preference. Am J Psychol 72: 487-502.

56. Palmer SE, Schloss K (2010) An ecological valence theory of human color preference. Proc Natl Acad Sci USA 107: 8877-8882.

57. Lind C (1993) Psychology of color: similarities between abstract and clothing color preferences. Clothing and Textiles Research Journal 12: 57-65.

58. Elliot AJ, Niesta D (2008) Romantic red: red enhances men's attraction to women. J Pers Soc Psychol 95: 1150-1164.

59. Guéguen N, Jacob C (2011) Color and cyber-attractiveness: red enhances men's attraction to women's internet personal ads. Color Res Appl 38: 309312

60. Clark N (2016) How food packaging color influences consumer behavior

61. Wright A (2012) Predicting responses to color. Colour Design: Theories and Applications. Woodhead Publishing, Oxford, UK

62. Wells D, McDonald C, Ringland J (2008) Color preferences in Gorillas (Gorilla gorilla gorilla) and chimpanzees (Pan troglodytes). J Comp Psychol 122: 213-219.

63. Crozier WR (1996) The psychology of colour preferences. Surface Coatings International 80: 577-585.

64. Wen F, Zuo B, Wu Y, Sun S, Liu K (2014) Red is romantic, but only for feminine females: sexual simorphism moderates red effect on sexual attraction. Evol Psychol 12: 719-735

65. Dixson AF (1998) Primate sexuality: comparative studies of the prosimians, monkeys, apes, and human beings. Oxford University Press, New York, USA.

66. Dixson AF, Herbert J (1977) Gonadal hormones and sexual behavior in groups of adult talapoin monkeys (Miopithecus talapoin). Horm Behav 8: $141-154$

67. Pazda A, Elliot A, Greitemeyer T (2012) Sexy red: perceived sexual receptivity mediates the red-attraction relation in men viewing women. $\mathrm{J}$ Exp Soc Psychol 48: 787-790.

68. Stankus T (2012) Reviews of science for science librarians: the evolutionary basis of human sexual attraction and competition for mates. Sci Tech Libr 31: $266-304$

69. Elliot A, Tracy J, Pazda A, Beall A (2013) Red enhances women's attractiveness to men: First evidence suggesting universality. J Exp Soc Psychol 49: 165-168.

70. Elliot A, Pazda A, Greitemeyer T (2013) Women's use of red clothing as a sexual signal in intersexual interaction. J Exp Soc Psychol 48: 599-602.

71. Stephen I, McKeegan A (2010) Lip colour affects perceived sex typicality and attractiveness of human faces. Perception 39: 1104-1110.

72. Stewart J (2013) ROY G. BIV: an exceedingly surprising book about color Bloomsbury, New York, USA

73. Faerm S (2010) Fashion: design course. Barron's, Hauppauge.

74. Finlay V (2014) The brilliant history of color in art. Getty Publications, Los Angeles, USA.

75. Finlay V (2004) Color: a natural history of the palette. Random House Publishing Group, New York, USA.

76. Office for National Statistics (2016) Migration statistics quarterly report: May 2016. 
77. Perry AW (2007) Straight talk about cosmetic surgery. Yale University Press Health \& Wellness. Yale University Press, New Haven, USA.

78. Wolfe MG (2012) Fashion! Goodheart-Willcox Co, Tinley Park, USA.

79. Biddle-Perry G, Cheang S (2008) Hair: styling, culture and fashion Bloomsbury Academic, New York, USA.

80. Breward C (2003) Fashion: Oxford history of art. Oxford University Press, New York, USA.

81. Riordan T (2004) Inventing beauty: a history of the innovations that have made us beautiful. Broadway Books, New York, USA.

82. Craik J (1994) The face of fashion: cultural studies in fashion. Routledge, London, UK.
83. Delio M (1994) Tattoo: the exotic art of skin decoration. St. Martin's Press, New York, USA.

84. Calderin J (2009) Form, fit, and fashion: all the details fashion designers need to know but can never find. Rockport Publishers, Beverly, USA.

85. Issett R (2007) Print, pattern and colour for paper and fabric. Batsford, London, UK.

86. Rigg B (2006) Colour description/specification systems. Total colour management in textiles. Woodhead Publishing Ltd, Cambridge, UK.

87. Sullivan C (2016) Investigation into color apparel as a feature of heterosexual attraction. Master of Science by Research in Polymers, Colorants and Fine Chemicals, University of Leeds, UK.

\section{Author Affiliations}

${ }^{1} 325$ N. Water St., Batavia, Illinois 60510, United States of America

${ }^{2}$ Department of Colour Science, School of Chemistry, Leeds University, UK

Submit your next manuscript and get advantages of SciTechnol submissions

* 80 Journals

* 21 Day rapid review process

* 3000 Editorial team

* 5 Million readers

* More than 5000

* Quality and quick review processing through Editorial Manager System

Submit your next manuscript at • www.scitechnol.com/submission 